\title{
Suppression of parasitic resonance in piezoresistively transduced longitudinal mode MEMS resonators
}

\author{
Jize Yan, Angel T-H. Lin, and Ashwin A. Seshia \\ Department of Engineering, University of Cambridge, Cambridge, United Kingdom
}

\begin{abstract}
This paper demonstrates the suppression of parasitic resonance in a piezoresistively transduced [1-3] longitudinal mode MEMS resonator, wherein beams are electrostatically excited in a combined extensional mode with an associated frequency- $Q$ product of $3.28 \times 10^{12}$. The response of the beam is sensed using both capacitive and piezoresistive transduction principles. The resonator consists of six parallel beams linked to a central anchor and a pair of symmetrical parallel beams that force the beams to vibrate in-phase. The mode suppression in the resonator is compared with other structures by finite element analysis (FEA). The relative distribution of strain energies in both the resonant structure and anchors [5] and in both primary and secondary directions of vibration are proposed as figures of merit to compare this device to previously reported longitudinal mode beam resonators $[2,4,6,7]$. The design optimization of longitudinal mode beam resonators is also discussed.
\end{abstract}

Keywords-Piezoresistive sensing, longitudinal mode, MEMS resonators, parasitic resonance;

\section{INTRODUCTION}

Silicon-based MEMS resonators have emerged as a potential candidate for applications relating to timing references, frequency selective signal processing and sensors due to their small size and potential for integrability with electronics. However, the efficiency of integrable transducer technologies and the presence of capacitive and motional parasitic elements has limited much of the applicability to the $\mathrm{HF}$ band and there are significant application drivers to scale operation to VHF and beyond.

Capacitively sensed silicon longitudinal bulk mode thinbeam MEMS resonators have demonstrated high quality factors but the performance is limited by the transduction area on each side of the beam [7]. Sandwich [4] and lateral bulk [6] resonators involve the combined longitudinal extension of parallel connected beams allowing for enhanced transduction area for the same operating frequency. However, flexural mode induced parasitic mechanical resonances can lead to energy loss from the primary resonant mode and limit device performance. Furthermore, capacitively actuated and piezoresistively sensed silicon MEMS resonators have been presented as a means for scaling to high frequencies with a 1.1 $\mathrm{GHz}$ fundamental mode silicon MEMS resonator demonstrated in a SOI process [1]. In this case, the resonator topology was modified to accommodate piezoresistive transduction also resulting in the coupling to parasitic mechanical resonances.

This paper demonstrates a novel piezoresistively transduced longitudinal mode MEMS resonators with the suppression of parasitic vibration modes, wherein beams are electrostatically excited in a combined extensional mode. A damping model was presented earlier to predict energy loss to the anchor by comparing the strain energy in the resonators to that in the anchor for a given mode shape [5]. The model has been extended in this paper to investigate the orientation distribution of the vibration energy for single degree-of-freedom longitudinal mode resonators. The paper utilizes this approach to compare the coupling of vibration energy into orthogonal directions for the longitudinal mode resonators previously reported $[2,4,6,7]$. FEA enhanced design optimization is also discussed.

Section II introduces the experimental results on resonator characterization and a comparison of transduction mechanisms. Section III discusses mode suppression and FEA assisted energy methods to compare various longitudinal mode resonators. Section IV uses the analysis principles and figures of merit derived in section III to propose design optimization of longitudinal mode resonators.

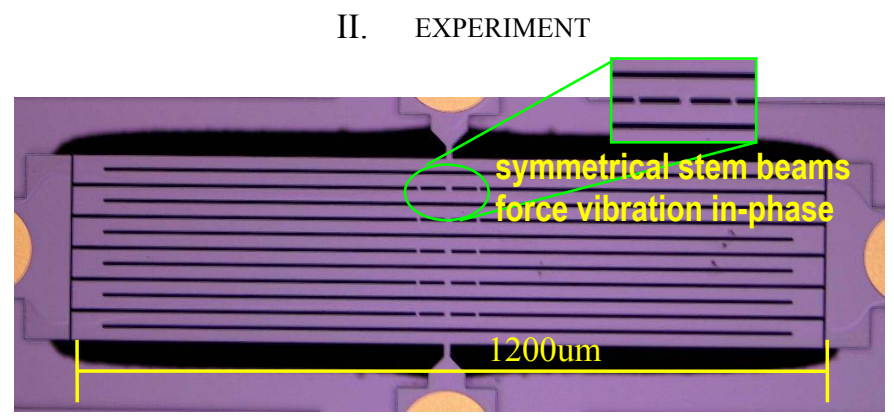

Figure 1. Optical micrograph of the coupled longitudinal mode resonators utilizing piezoresistive sensing.

The device under test is a $3.5 \mathrm{MHz}$ resonator operated in the bulk longitudinal mode, wherein the resonator undergoes an extension and contraction symmetrically along the longitudinal axis. The resonator consists of 6 pairs of parallel beams, which are linked by a central beam and two symmetrically situated thin beams running along the transverse direction as illustrated in Fig. 1. The resonator is fabricated in a commercial SOI MEMS foundry process (MEMSCAP), with lateral electrodes situated on both sides of the structure for capacitive excitation and detection. The transduction gap is designed to be $2 \mu \mathrm{m}$. The resonator dimension parameters are listed in Table 1 . The symmetrical parallel beams that run along the transverse direction connect the longitudinal beams and force them to vibrate in-phase. Parasitic resonances are suppressed in this construction. The structure is anchored to the substrate via 
straight beams connecting to anchors located on two opposite sides of the coupled parallel beams.

TABLE I. DIMENSIONS OF THE RESONATOR WITH STRAIGHT ANCHORS

\begin{tabular}{|l|c|}
\hline Parameter (units) & Value \\
\hline Number of beams & 12 \\
\hline Spacing between beams $(\mu \mathrm{m})$ & 5 \\
\hline Length of a single beam $(\mu \mathrm{m})$ & 1200 \\
\hline Width of a single beam $(\mu \mathrm{m})$ & 20 \\
\hline Length of straight anchor $(\mu \mathrm{m})$ & 40 \\
\hline Width of straight anchor $(\mu \mathrm{m})$ & 20 \\
\hline Width of the central link beam $(\mu \mathrm{m})$ & 20 \\
\hline Width of the symmetical thin stem beams $(\mu \mathrm{m})$ & 5 \\
\hline
\end{tabular}

An electrical connection is provided to each anchor of the resonator body. This enables the capacitive and piezoresistive sensing configurations used in this work. The resonator was characterized in a custom-built vacuum chamber at pressures below 10mTorr. The electrical transmission of each device was measured using a network analyzer. The same voltages are applied to the other longitudinal more resonators. Q values have been extracted from measurements of the S21 transmission using the procedure described in [5].

In the two port configuration (figure 2), the resonator body is connected to a DC bias and the electrodes are split in two pairs, one used for capacitive driving and the other for capacitive sensing to reduce the feedthrough through the direct overlap capacitor between the actuator electrode and the sensing port [3]. However, feedthrough currents can still exist via the substrate, package and bond wires, which can mask the motional current as shown in the measured transmission response in Fig. 3.

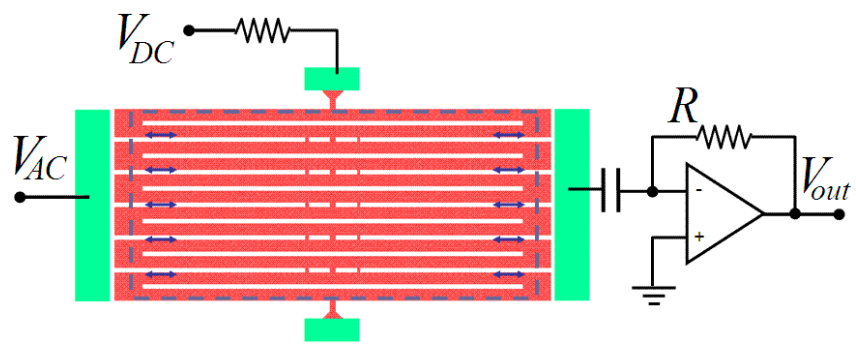

Figure 2. Schematic showing measurement setup for two port capacitive actuation and sensing.

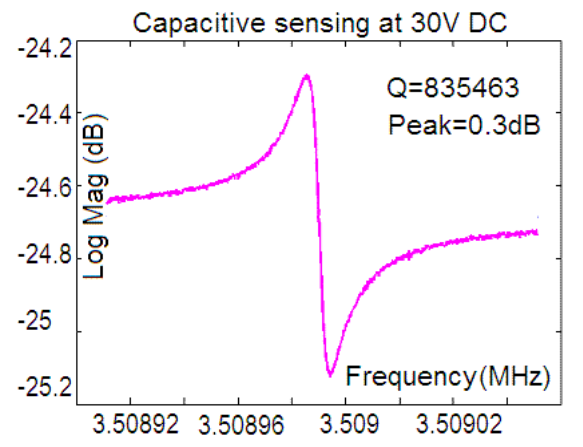

Figure 3. Measured electrical transmission of the coupled longitudinal mode beam microresonators with two port capacitive acutation and sensing.
The resonator frequency response is also measured through the variation in the electrical resistance of the vibrating structure due to the piezoresistive effect [3]. This method has been previously shown to be a promising alternative for increasing the amplitude of the motional signal relative to the capacitive feedthrough for particular topologies of bulk mode resonators [1]. The open-loop transmission measurements for the resonator are characterized utilizing the setup schematic shown in Fig. 4. The resonator demonstrates an impressive quality factor of 0.936 million at $3.5 \mathrm{MHz}$ using piezoresistive readout shown in figure 5. Also, by utilizing a recently reported second harmonic piezoresistive sensing approach [3], a Q of 6870 is observed in the air shown in Fig. 6. A clearly enhanced transmission response can be observed for piezoresitive sensing compared to capacitive sensing as expected.

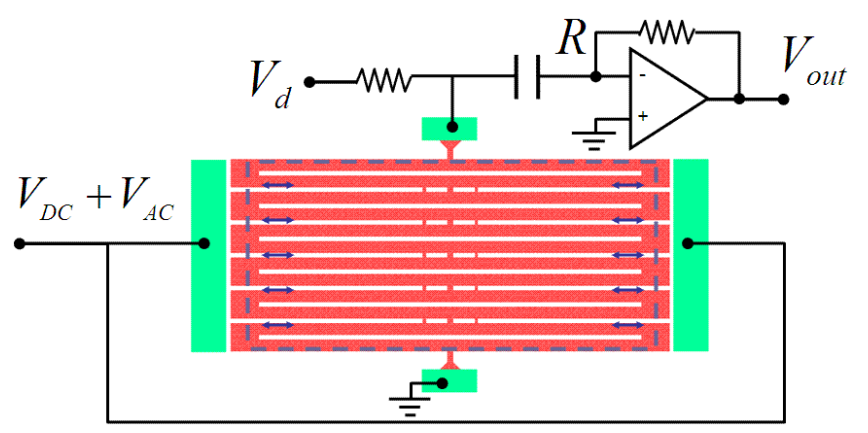

Figure 4. Schematic showing measurement setups for 2 nd harmonic drive and piezoresistive sensing.

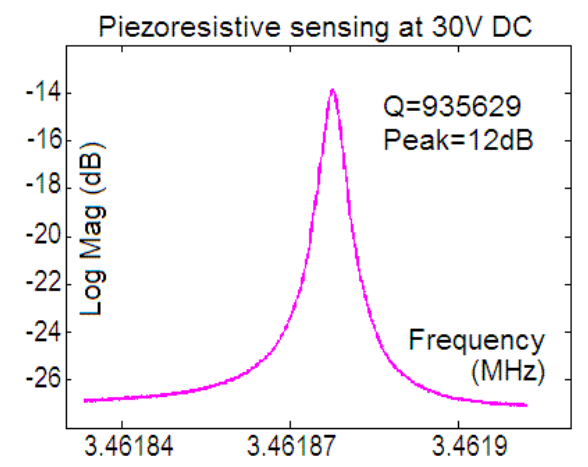

Figure 5. Measured electrical transmission of the coupled longitudinal mode beam microresonators utilizing piezoresistive sensing in vacuum.

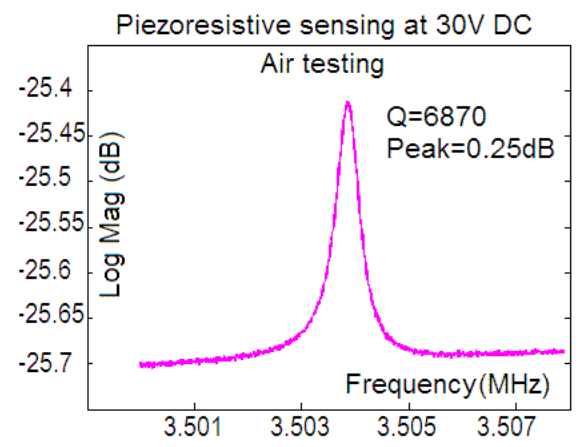

Figure 6. Transmission response of coupled longitudinal mode resonators measured in air. 


\section{MODELING AND SimUlation}

\section{A. Mode suppression}

Parasitic mechanical resonance in the coupled beam longitudinal mode resonators is suppressed and this can be ascertained from the measured frequency response of the resonators. Figure 7 shows the FEA mode shape of the coupled longitudinal mode resonators without the symmetrical stem beams shown in Fig. 1 but consisting of the central transverse beam connector. Without the mode suppression, the six parasitic resonances as shown in Fig. 7 will strongly influence the resonator transduction, in which the frequency of the parasitic resonances are closely spaced within the range from $3.474 \mathrm{MHz}$ to $3.476 \mathrm{MHz}$.
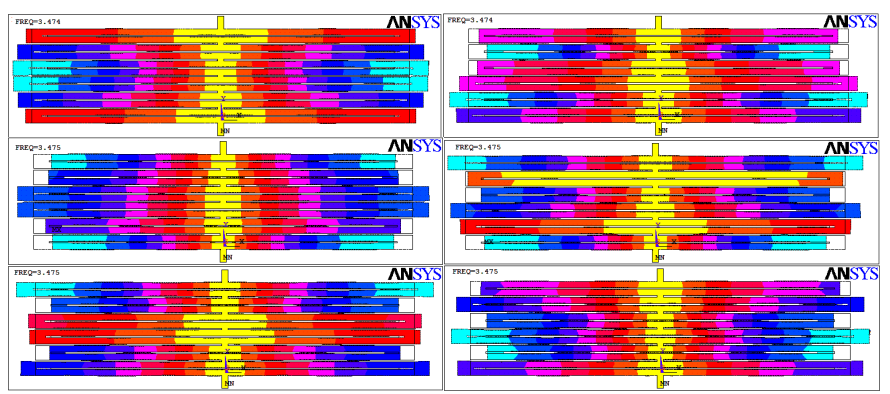

Figure 7. Mode shapes of coupled longitudinal mode resonators without suppression of the parasitic resonances.

\section{B. FEA computed stress distribution}

In order to compare the performance of the bulk longitudinal mode beam resonators, FEA computed stress distribution is illustrated in Fig. 8. This includes calculations for longitudinal bulk mode beam resonators previously reported $[2,4,6,7]$. The dimensions utilized in the FEA simulation are obtained from the literature and the material properties used are those for single-crystal silicon.

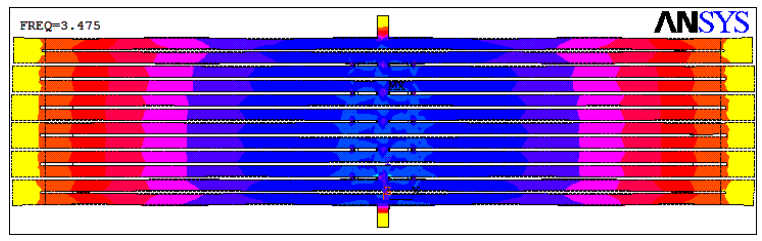

(a) Mode suppressed coupled beam longitudinal mode resonators (this work)

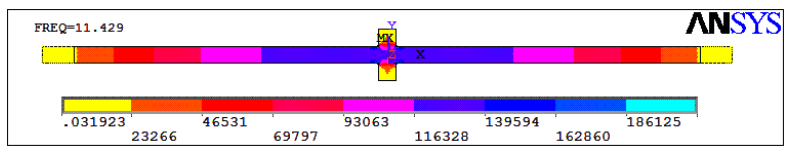

(b) VTT longitudinal mode resonators (reference [7])

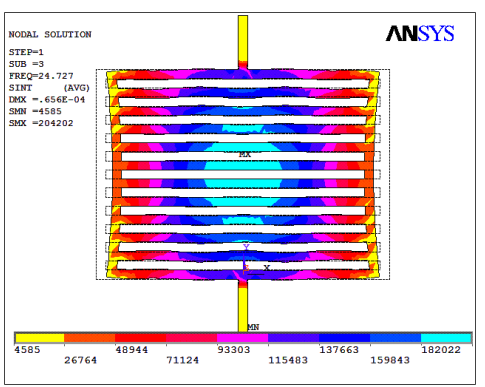

(c) EPFL FBR 115 resonators (reference [6])

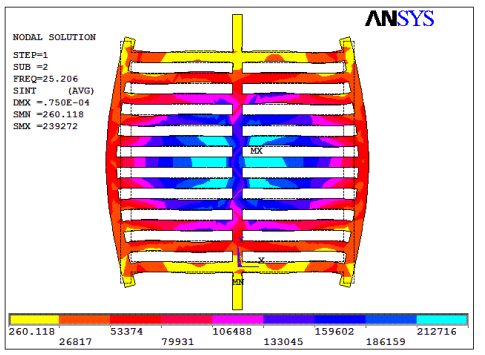

(d) Sandwich longitudinal mode resonators (reference [4])

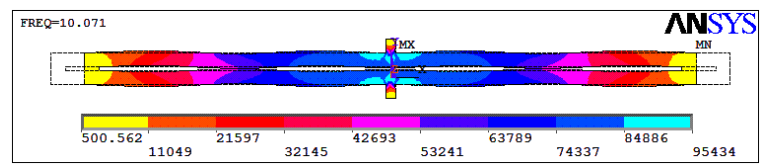

(e) Piezoresistively sensed longitudinal mode resonators (reference [2])

Figure 8. FEA computed stress distribution for various bulk longitudinal mode beam resonators.

\section{Energy stored in entire structure over anchors}

The anchor limited Q of a resonator may be approximated by calculating the ratio of the maximum total strain energy in the resonator relative to the strain energy in the anchor and connecting stem. This approximation assumes that a fraction of the strain energy in the stem and anchor region is lost during vibration, thus defining the anchor limited Q [5].

\section{Energy stored in $x$ direction over $y$ direction}

Bulk longitudinal mode beam resonators are ideally vibrating along one direction only. Therefore, the vibrational energy coupled in an orthogonal direction is an important figure of merit defining its energy handling ability.

\section{E. Comparison of longitudinal mode resonators}

TABLE II. COMPARISON OF LONGITUDINAL MODE RESONATORS

\begin{tabular}{|l|c|c|c|c|c|}
\hline Topologies & $\mathrm{E}_{\mathrm{T}} / \mathrm{E}_{\mathrm{A}}$ & $\mathrm{E}_{\mathrm{X}} / \mathrm{E}_{\mathrm{Y}}$ & \multicolumn{2}{|c|}{ Freq. $(\mathrm{MHz})$} & \multirow{2}{*}{ Test Q } \\
\cline { 4 - 5 } & & & Test & FEA & \\
\hline This work & 90950 & 4669 & 3.50 & 3.47 & $9.3 \mathrm{E} 5$ \\
\hline VTT bulk [7] & 964 & 24909 & 11.7 & 11.4 & $1.8 \mathrm{E} 5$ \\
\hline EPFL-FBR115[6] & 22962 & 305 & 24.6 & 24.7 & $7.2 \mathrm{E} 4$ \\
\hline Sandwich [4] & 20034 & 38.7 & 25.1 & 25.2 & $1.3 \mathrm{E} 4$ \\
\hline NXP bulk [2] & 27760 & 624 & 10.5 & 10.7 & $1.25 \mathrm{E} 5$ \\
\hline
\end{tabular}

$\mathrm{E}_{\mathrm{T}} / \mathrm{E}_{\mathrm{A}}$ : FEA calculated ratio of strain energy stored in resonator to the anchors.

$\mathrm{E}_{\mathrm{X}} / \mathrm{E}_{\mathrm{Y}}$ : FEA calculated strain energy stored in $x$ direction relative to the $y$ direction. 
Table II compares the performance of various longitudinal mode beam resonators. Resonator dimensions and results on frequency and $\mathrm{Q}$ are obtained from literature $[2,4,6,7]$.

\section{DISCUSSION AND DESIGN OPTIMIZATION}

The metrics, $\mathrm{E}_{\mathrm{T}} / \mathrm{E}_{\mathrm{A}}$ and $\mathrm{E}_{\mathrm{X}} / \mathrm{E}_{\mathrm{Y}}$, are used to develop a design optimization and sizing approach for longitudinal mode beam resonators. This approach is captured graphically in Figs. 9-11. For VTT bulk mode resonators [7], the metrics decrease as a function of W/L, and an optimum is achieved when the widthto-length ratio is small. Placing the anchor at the centre of the two sides of the resonators will prevent motion in the $y$ direction. However, the sandwich [4] and EPFL FBR [6] resonators show a different scaling behavior as shown in Fig. 10. Figure 11 shows the scaling behavior for the coupled beam longitudinal mode resonators described in this work. As shown, increasing the number of coupled beams will not degrade performance, because both the figures of merit and stored energy are enhanced by increasing transduction area.

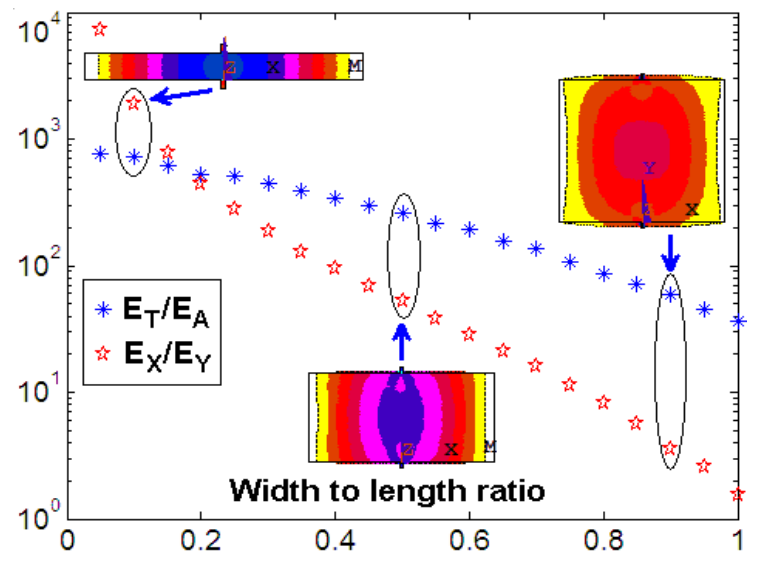

Figure 9. FEA simulation of anchor energy ratio and orientation energy ratio of VTT longitudinal mode resonator with variable width to length ratio

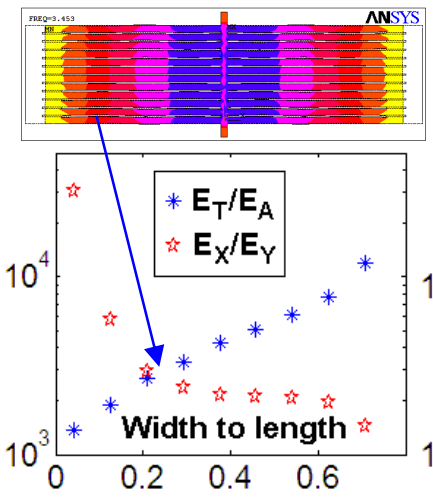

(a) Sandwich type resonator

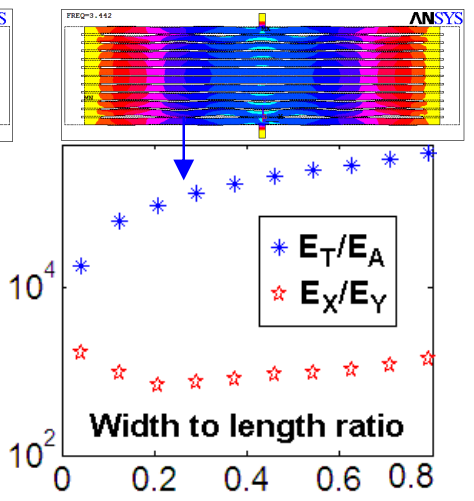

(b) EPFL FBR Type resonator
Figure 10. FEA simulation of anchor energy ratio and orientation energy ratio of (a) sandwich resonator and (b) EPFL FBR resonators

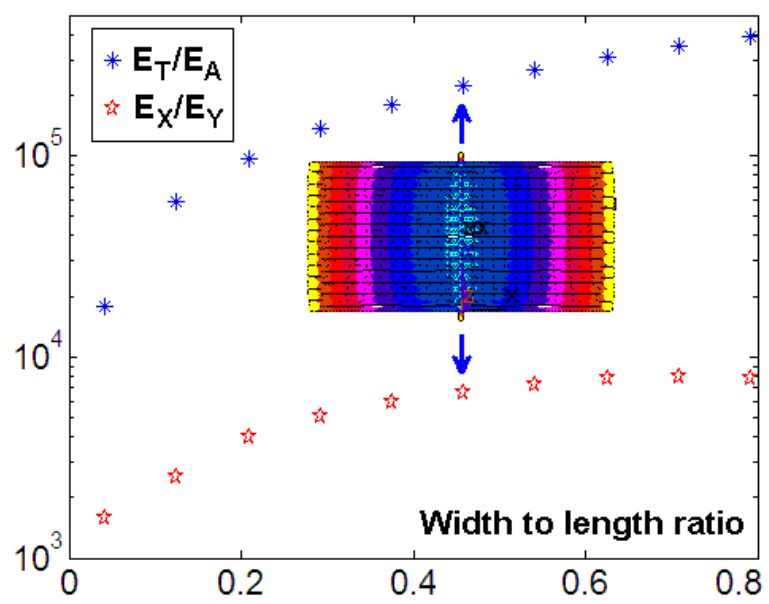

Figure 11. FEA simulation of anchor energy ratio and orientation energy ratio of the coupled beam longitudinal mode resonator reported in this work with variable $\mathrm{W} / \mathrm{L}$

\section{CONCLUSIONS}

A novel piezoresistively-transduced and spurious mechanical mode suppressed longitudinal mode resonator is demonstrated with an associated frequency-Q product of $3.28 \times$ $10^{12}$. This topology allows for the scaling of the resonator transconductance at high $\mathrm{Q}$ by combining an arrayed beam approach while simultaneously rejecting spurious mechanical modes. Figures of merit relating factors of anchor loss and orientation distribution of the vibrational energy in longitudinal mode resonators are benchmarked versus previous work.

\section{REFERENCES}

[1] J.T.M. van Beek, G.J.A. Verheijden, G.E.J. Koops,K.L. Phan,C. van der Avoort, J. van Wingerden, D.E. Badaroglu, J.J.M. Bontemps, 'Scalable $1.1 \mathrm{Ghz}$ fundamental mode piezo-resistive silicon MEMS resonators.' Proc. IEDM 2007, pp. 411-414.

[2] J.T.M. Van Beek, P.G. Steeneken, and B. Giesbers, "A $10 \mathrm{MHz}$ piezoresistive MEMS resonator with high Q," Proc. IEEE Int. FCS 2006, pp. $475-480$.

[3] A. T-H. Lin, J. E-Y. Lee, J. Yan, A. A. Seshia, 'Enhanced Transduction Methods for Electrostatically Driven MEMS Resonators', Proc. Transducers 2009, pp. 561-564.

[4] J.Yan, A.A. Seshia, P.G. Steeneken, J.T.M van Beek, 'A silicon MEMS bulk mode sandwich resonator,' Proc. MME 2006, pp.217-220.

[5] J. E-Y. Lee, J. Yan, A. A.Seshia, 'Anchor limited Q in flexural mode resonators', Proc. IEEE IUS 2008, pp. 2213-2216.

[6] D. Grogg, H. C. Tekin, N. D. Ciressan-Badila, D. Tsamados, M. Mazza, and A.M. Ionescu, 'Bulk Lateral MEM Resonator on Thin SOI With High Q-Factor,' JMEMS, vol.18, no.2, 2009, pp.466-479.

[7] T. Mattila, J. Kiihamäki, T. Lamminmäki, O. Jaakkola, P. Rantakari, A. Oja, H. Seppä, H. Kattelus, I. Tittonen, ' $12 \mathrm{MHz}$ micromechanical bulk acoustic mode oscillator,' Sens. Actuators A, vol. 101, no. 1-2, pp. 1-9, Sept. 2002 